\title{
Anisotropic Strength of Granular Material Considering Fabric Evolution
}

\author{
Yang Liu ${ }^{\mathrm{a}^{*}}$ (1) \\ Duo Zhang \\ Xiaoxiao Wang ${ }^{\mathrm{a}^{*}}$ \\ Pengqiang $\mathrm{Yu}^{\mathrm{a}}$
}

\section{Wei Hua}

a Department of Civil Engineering, University of Science and Technology, Beijing, 100083, China. E-mail: yangliu@ustb.edu.cn, 17801093732@163.com,yu_pengqiang@163.com,531749355@qq.com

b Wuhan Institute of Comprehensive Transportation CO. LTD, Wuhan, China. E-mail: tmyxzd@ hotmail.com

* Corresponding author:

http://dx.doi.org/10.1590/1679-78255475

\begin{abstract}
The effect of fabric anisotropy on strength has not been considered in most strength criterions for granular material. Some criterions can describe the variation of material strength with stress-induced anisotropy, but micro-mechanism and physical meanings are undefined. Some criterions consider the effect of fabric anisotropy, but the evolution of fabric is ignored during loading by assuming a constant fabric tensor. Based on strength mechanical characteristics of granular material, in this paper, the relationship between macro stress and micro contact force of granular material is derived by micromechanics. Then, the concept of true stress tensor is proposed. An anisotropic strength criterion of granular material considering fabric evolution is established and its applicability is validated by comparing with test results for different granular materials. The analysis results indicate that the proposed anisotropic criterion can be utilized to describe the strength feature of anisotropic granular materials, which gives a way for the cause analysis of the strength of granular materials from the perspective of Microscopic mechanism.
\end{abstract}

\section{Keywords}

Granular material; Anisotropy; Fabric; Strength.

\section{INTRODUCTION}

Strength problem is always a focus in granular mechanics. Numerous strength criteria for granular material have been developed so far, such as the Mohr-Coulomb (M-C) strength criterion, Matsuoka-Nakai (M-N) criterion (Matsuoka and Nakai, 1974), Lade-Duncan (L-D) criterion (Lade and Duncan, 1975) and so on. However, these theories have more or less limitations. For example, $\mathrm{M}-\mathrm{C}$ criterion does not take into account the effect of middle principal stress and the deviatoric shape is hexagon; $\mathrm{M}-\mathrm{N}$ strength criterion predicts the same strength with $\mathrm{M}-\mathrm{C}$ criterion for both triaxial compression (TC) and triaxial extension (TE)condition, although the effect of middle principal stress is taken into account; While the strength of L-D criterion under TE is higher than that of the other two. Additionally, from the perspective of micromechanics, M-N and L-D strength criteria can reflect the effect of induced anisotropy to some extent. None of above three strength criteria, however, can reflect the change of strength surface in complex stress for initial anisotropic 
material, for their expressions only contain a strength parameter, internal friction angle obtained from triaxial compression test.

It is a fact that the granular materials (i.e. sand) have initial anisotropy due to preferred orientation of particles and deposition. Strong influences of anisotropy on shear behaviors and peak friction angle of sand have been observed by numerous experiments including true triaxial tests (Rodriguez and Lade, 2013), hollow cylinder tests (Yang et al., 2015), plane strain tests (Oda et al, 1978) and DEM simulations (Lü et al, 2017). Therefore, on the basis of the above classical strength criteria, several anisotropic strength criteria of granular material have been proposed by different scholars. Yoshimine (2006) proposed the generalized Coulomb's criterion for three-dimensional stress conditions based on the concept of reference mobilized plane (RMP) and further analyzed the change regulation of failure envelope with general directions of RMP. Similarly, Shao et al. (2015) proposed a strength criterion based on the $\sqrt[3]{\sigma}$ spatially mobilized plane, on which the shear stress ratio is constant. Yao and Kong (2012) proposed a three-dimensional cross-anisotropic strength criterion by combining the SMP strength criterion with the transformed stress tensor, which is a function of cross-anisotropic peak strength and stress tensor. Lü et al (2016) established a cross-anisotropic strength criterion by introducing the Lode dependence and fabric tensor into the 3D M-C criterion. The classical isotropic failure criteria was generalized to anisotropic criteria by Tian and Yao (2017) using an anisotropic transformed stress method. Cao et al (2016) extended the L-D criterion to be an anisotropic L-D criterion by introducing a strength variable $\Lambda$ related to stress tensor and the orientation of bedding plane and the obtained formula can also generalized the isotropic $\mathrm{M}-\mathrm{C}$ and $\mathrm{M}-\mathrm{N}$ criterion to become anisotropic. In addition, there are also various cross-isotropic strength criteria for geomaterials to characterize the stress-strain-strength anisotropy by introducing variables that include the information of material fabric and external loading into classical isotropic strength criteria. (Xiao et al., 2012, Lade, 2007, Kong et al., 2013). Meanwhile, many anisotropic failure criteria for granular medium was established by combining fabric and stress tensor (Pietruszczak and Mroz, 2000; Tobita and Yanagisawa, 1992; Tobita, 1993; Schweiger et al., 2009; Gao et al., 2010; Liu, 2013.

However, the existing strength criteria mentioned above do not fully incorporate the effect of fabric. For example, $\mathrm{M}-\mathrm{C}$ criterion completely ignores the effect of fabric anisotropy on strength. Although L-D criterion can describe the change of strength with induced anisotropy for isotropic material, it doesn't explain the mechanism from the microscopic microcosmic level. On the other hand, some criteria taking into account the effect of initial fabric anisotropy often assume that the fabric tensor remains constant during loading, which is not complete in theory yet. (i.e. Tobita and Yanagisawa, 1992; Tobita, 1993; Liu, 2013).

In this paper, the relationship between macroscopic stress and microscopic contact force of granular material is derived by micromechanical method at first and the concept of true stress is then proposed. After that, a novel anisotropic strength criterion considering fabric evolution is proposed for granular medium by introducing the fabric evolution formula into the true stress tensor. Finally, the applicability of the proposed strength criterion was validated by comparing with experimental results for different granular materials.

\section{ANALYSIS OF CONTACT FORCE IN GRANULAR PARTICLES}

\subsection{Inter-Partical Contact Force and True Stress}

The mean stress in a representative volume $V$ can be written in terms of the inter-particle contact forces (Christofferson et al., 1981; Rothenburg and Selvadurai, 1981):

$\sigma_{i j}=\frac{1}{V} \sum_{c} f_{j} l_{i}$

where $l_{i}=r_{i}^{n c}-r_{i}^{m c}$ is the branch vector connecting the center of two particles $m$ and $n$; $f_{j}$ is the contact force between particles; $V$ is the representative volume. In order to calculate the inter-particle contact force $f_{j}$, Chang and Gao (1996) proposed a static hypothesis:

$f_{j}=\sigma_{i j} A_{i k} l_{k}$

where $A_{j k}$ is a second-order tensor, and it can be written in terms of the fabric tensor $F_{i k}$ 
$A_{i k}=\frac{V}{4 \bar{r}^{2} N}\left(F_{i k}\right)^{-1}$

where $\bar{r}$ is the average length of branch vector; $F_{i k}$ is the fabric tensor describing the spatial arrangement of granular particles, which is usually defined as:

$F_{i k}=\frac{1}{N} \sum_{k=1}^{N} n_{i} n_{k}$

where $N$ is the total number of contacts; $n_{i}, n_{k}$ are the unit contact normal.

Substituting Eq.(3) into Eq. (2), we obtain

$f_{j}=\frac{V}{4 \bar{r}^{2} N} \sigma_{i j}\left(F_{i k}\right)^{-1} l_{k}$

A new second-order tensor $T_{i j}$, named "true stress tensor", is then defined here

$T_{i j}=\frac{V}{4 \bar{r}^{2} N} \sigma_{k i}\left(F_{k j}\right)^{-1}$

Then Eq.(5) can be rewritten as

$f_{j}=T_{i j} l_{i}$

Eq. (7) indicates that the inter-particle contact force is the projection of second-order tensor $T_{i j}$ on the branch vector, which is similar to the projection rule proposed by Jenkins and Strack (1993). The projection rule is derived from the mean field value theory (MFV), which suggests that the local microscopic variables can be calculated by the projection of the gradient of the macroscopic variables on corresponding branch vectors.

Liu et al. (2014) pointed out that the strength of granular assembly under external load is supported by two parts: one is "true stress", which controls the strength and deformation of granular material, and the other is anisotropic structure of granular assembly. Thus, it is significant to introduce the true stress tensor into anisotropic strength of granular material. First, the projection of true stress tensor on branch vector is the corresponding contact force, therefore it plays a bond role between the macro- and micro- mechanical properties of the specimen, which is of great significance in micromechanical study. Second, the true stress tensor is beneficial to the reason analysis on granular material's strength, for it implies the frictional effect in the assembly.

\subsection{INTER-PARTICLE CONTACT FORCE CALCULATION}

Assuming the co-axiality between the principal axes of stress tensor and fabric tensor, the inter-particle contact force's normal component can be given as:

$f_{n}=f_{i} n_{i}=\frac{V}{2 r N}\left(\frac{\sigma_{11}}{F_{11}} n_{1}^{2}+\frac{\sigma_{22}}{F_{22}} n_{2}^{2}+\frac{\sigma_{33}}{F_{33}} n_{3}^{2}\right)$

The tangential component is deduced as:

$f_{s}=\frac{V}{2 r N} \sqrt{\left(\frac{\sigma_{11}}{F_{11}}-\frac{\sigma_{22}}{F_{22}}\right)^{2} n_{1}^{2} n_{2}^{2}+\left(\frac{\sigma_{22}}{F_{22}}-\frac{\sigma_{33}}{F_{33}}\right)^{2} n_{2}^{2} n_{3}^{2}+\left(\frac{\sigma_{11}}{F_{11}}-\frac{\sigma_{33}}{F_{33}}\right)^{2} n_{1}^{2} n_{3}^{2}}$ 
Thus, the ratio of contact force (tangential contact force to normal contact force) can be written as:

$\frac{f_{s}}{f_{n}}=\frac{\sqrt{\left(\frac{\sigma_{11}}{F_{11}}-\frac{\sigma_{22}}{F_{22}}\right)^{2} n_{1}^{2} n_{2}^{2}+\left(\frac{\sigma_{22}}{F_{22}}-\frac{\sigma_{33}}{F_{33}}\right)^{2} n_{2}^{2} n_{3}^{2}+\left(\frac{\sigma_{11}}{F_{11}}-\frac{\sigma_{33}}{F_{33}}\right)^{2} n_{1}^{2} n_{3}^{2}}}{\frac{\sigma_{11}}{F_{11}} n_{1}{ }^{2}+\frac{\sigma_{22}}{F_{22}} n_{2}^{2}+\frac{\sigma_{33}}{F_{33}} n_{3}^{2}}$

Since

$$
\sigma_{11} / F_{11}=\frac{4 \bar{r}^{2} N}{V} T_{11}, \sigma_{22} / F_{22}=\frac{4 \bar{r}^{2} N}{V} T_{22}, \sigma_{33} / F_{33}=\frac{4 \bar{r}^{2} N}{V} T_{33}
$$

obtained from Eq.(6), thus the contact force ratio can also be rewritten as:

$$
\frac{f_{s}}{f_{n}}=\frac{\sqrt{\left(T_{11}-T_{22}\right)^{2} n_{1}^{2} n_{2}^{2}+\left(T_{22}-T_{33}\right)^{2} n_{2}^{2} n_{3}^{2}+\left(T_{11}-T_{33}\right)^{2} n_{1}^{2} n_{3}^{2}}}{T_{11} n_{1}{ }^{2}+T_{22} n_{2}{ }^{2}+T_{33} n_{3}^{2}}
$$

\subsection{CONTACT SLIDING AND GENERALIZED COULOMB CRITERION}

Eq. (11) shows clearly that contact force ratio is not only related to stress, but also fabric representing the orientation of contacts. The contact force ratio varies in different directions. In the simple slip model, the contact failures and relative slip occur simultaneously between particles when the contact force ratio is beyond a certain limit.

If all contacts slip simultaneously in a certain direction, the contact force ratio in this direction will reach the maximum $\left(f_{s} / f_{n}\right)_{\text {peak }}$ which satisfies Eq. (11). The macroscopic peak stress of the material is dependent on the fabric, orientation of contacts and peak contact force ratio. It is confirmed that the preferred slip direction of contacts is that of macroscopic slip surface if all contacts slip simultaneously in the same direction. Specially, when the granular material is in a two-dimensional isotropic state, the normal vector of preferred slip surface of contacts is given by:

$\frac{n_{1}}{n_{3}}=\sqrt{\frac{\sigma_{33}}{\sigma_{11}}}, n_{2}=0$

So, Eq. (10) can be simplified as

$\left(\frac{f_{s}}{f_{n}}\right)_{\text {peak }}=\frac{\sigma_{11}-\sigma_{33}}{2 \sqrt{\sigma_{11} \sigma_{33}}}=\tan \varphi_{\mu}$

Eq. (13) is in accordance with the classical Mohr-Coulomb criterion (Taylor, 1948; Mitchell and Soga. ,2005), but it is noted that $\varphi_{u}$ is the friction angle of particle, not of the friction angle of the assemblies.

A generalized Coulomb criterion based on reference mobilized plane proposed by Yoshimine (2006) can be expressed as:

$\frac{\tau_{R M P}}{\sigma_{R M P}}=\frac{\sqrt{\left(\sigma_{11}-\sigma_{22}\right)^{2} n_{1}{ }^{2} n_{2}{ }^{2}+\left(\sigma_{22}-\sigma_{33}\right)^{2} n_{2}{ }^{2} n_{3}{ }^{2}+\left(\sigma_{11}-\sigma_{33}\right)^{2} n_{1}{ }^{2} n_{3}{ }^{2}}}{\sigma_{11} n_{1}{ }^{2}+\sigma_{22} n_{2}{ }^{2}+\sigma_{33} n_{3}{ }^{2}}$

By comparing the Eq. (11) with Eq. (14), it is found that the proposed strength criterion will reduce to the generalized Coulomb strength criterion proposed by Yoshimine if an initially isotropic material is considered and its fabric keeps constant in the process of loading. 


\section{ANISOTROPIC STRENGTH OF GRANULAR MATERIAL CONSIDERING FABRIC EVOLUTION}

\subsection{ANISOTROPIC STRENGTH CRITERION}

As mentioned above, Eq. (11) indicates that the slip surface changes with the evolution of fabric during loading, thus the proposed criterion considering fabric evolution is actually the general form of the generalized Coulomb criterion by Yoshimine. In addition, the true stress tensor, not stress tensor used in the generalized Coulomb criterion, is adopted in Eq. (11) to analyze the strength anisotropy in an isotropic stress space. Therefore, the expression of the orientation of slip surface should also be expressed by true stress tensor:

$\left(\frac{n_{i}}{n_{j}}\right)^{2}=\left(\frac{T_{j}}{T_{i}}\right)^{m}$

Where, $m$ is a parameter related to orientation of failure surface. It is noted that Eq (15) will reduce to the MatsuokaNakai (M-N) criterion for isotropic materials and $m=1$.

In the generalized Coulomb criterion proposed by Yoshimine (2006), the direction of the failure surface is determined by fitting the peak contact force ratio of various stress paths on the $\pi$ plane. In this paper, however, the peak contact force ratio of initially isotropic materials can be easily obtained by substituting Eq. (15) into Eq. (11):

$\left(\frac{f_{s}}{f_{n}}\right)_{\text {peak }}=\frac{\sqrt{\frac{\left(T_{11}-T_{22}\right)^{2}}{T_{11}^{m} T_{22}^{m}}+\frac{\left(T_{22}-T_{33}\right)^{2}}{T_{22}^{m} T_{33}^{m}}+\frac{\left(T_{11}-T_{33}\right)^{2}}{T_{11}^{m} T_{33}^{m}}}}{T_{11}^{1-m} T_{22}^{1-m} T_{33}^{1-m}}$

For the same material, the peak contact force ratio is a material constant independent of the middle principal stress parameter $b=\left(\sigma_{2}-\sigma_{3}\right) /\left(\sigma_{1}-\sigma_{3}\right)$, implying that the peak contact force ratio under triaxial compression state should be identical to that in triaxial tensile state, which can be written as:

$\left(\frac{f_{s}^{T C}}{f_{n}^{T C}}\right)_{\text {peak }}=\left(\frac{f_{s}^{T E}}{f_{n}^{T E}}\right)_{\text {peak }}$

\subsection{ANISOTROPIC STRENGTH CRITERION CONSIDERING FABRIC EVOLUTION}

According to the above analysis, the anisotropic strength criterion of the granular material expressed by true stress is obtained as follows,

$$
f\left(T_{11}, T_{22}, T_{33}, H_{T}\right)=\sqrt{\frac{\left(T_{11}-T_{22}\right)^{2}}{T_{11}^{m} T_{22}^{m}}+\frac{\left(T_{22}-T_{33}\right)^{2}}{T_{22}^{m} T_{33}^{m}}+\frac{\left(T_{11}-T_{33}\right)^{2}}{T_{11}^{m} T_{33}^{m}}}-H_{T}\left(T_{11}^{1-m}+T_{22}^{1-m}+T_{33}^{1-m}\right)=0
$$

Where $H_{T}$ is the peak contact force ratio in Eq. (16).

As we know, the fabric evolution of the granular material will occur to adapt to the new stress state in the process of loading (Nemat-Nasser, 2000; Wan and Guo, 2001). Thus, it is necessary to obtain a fabric evolution law of granular materials in that the true stress tensor includes the fabric tensor in Eq. (18). Based on numerous true triaxial numerical tests with discrete element method, a fabric evolution law of granular material is proposed as follows:

$$
F_{i j}=\beta\left(\frac{\sigma_{i j}}{p}-1\right)+\left.F_{i j}\right|_{0}
$$

where $\beta$ is the parameter of fabric evolution, $p$ is the mean stress and $\left.F_{i j}\right|_{0}$ is the initial fabric. 
Parameter $\beta$ is assumed to be independent of $b, m$-value can be calculated directly for there is only an unknown $m$ in Eq. (16). After the friction angle and $m$-value are determined, the peak force ratio can be calculated from Eq. (16). Thus, the proposed anisotropic strength criterion of granular material considering evolution of fabric is expressed by Eq. (18) and Eq. (19).

\section{PARAMETER DETERMINATION}

\subsection{INITIAL FABRIC $\left.F_{i j}\right|_{0}$ AND FABRIC PARAMETER $\beta$}

Generally, the initial fabric of a specimen can be measured by micro- statistics method, as well as the peak fabric $F_{i j}^{p e a k}$ or critical fabric $F_{i j}^{c r}$. The peak friction angles $\varphi_{p}^{T C}, \varphi_{p}^{T E}$ or critical friction angles $\varphi_{c r}^{T C}, \varphi_{c r}^{T E}$ can be obtained by triaxial compression and triaxial extension tests, respectively. Hence, the fabric parameter $\beta$ can be derived from Eq. (18) as follow:

$$
\beta=\frac{1}{3} \sum_{i=1}^{3} \frac{F_{i j}^{\text {peak }(c r)}-\left.F_{i j}\right|_{0}}{\left(\sigma_{i j}-p\right) / p},(i=j)
$$

where $\left.F_{i j}\right|_{0}$ is the component of initial fabric; $F_{i j}^{\text {peak (cr) }}$ is the peak (critical) fabric component.

However, it should be noticed that the measurements of fabric in laboratory tests are practically complicated. In this case, the initial fabric and the parameter $\beta$ can also be empirically determined.

\subsection{PARAMETERS $m$ AND $H_{T}$}

The strength parameter $m$, representing the orientation of failure surface, can be obtained by combining TC and TE test results. The details are as follows.

First, Eq. (16) can be simplified as follow by multiplying both the top and the bottom by $T_{1}^{\frac{m}{2}} T_{2}^{\frac{m}{2}} T_{3}^{\frac{m}{2}}$ :

$$
H_{T}=\frac{\sqrt{\left(T_{1}-T_{2}\right)^{2} T_{3}^{m}+\left(T_{1}-T_{3}\right)^{2} T_{2}^{m}+\left(T_{2}-T_{3}\right)^{2} T_{1}^{m}}}{T_{1}^{1-\frac{m}{2}} T_{2}^{\frac{m}{2}} T_{3}^{\frac{m}{2}}+T_{1}^{\frac{m}{2}} T_{2}^{1-\frac{m}{2}} T_{3}^{\frac{m}{2}}+T_{1}^{\frac{m}{2}} T_{2}^{\frac{m}{2}} T_{3}^{1-\frac{m}{2}}}
$$

Then, substituting $k_{T i j}=T_{i} / T_{j}$ into Eq.(21), we thus have:

$$
\begin{aligned}
& H_{T}^{T C}=\frac{\sqrt{\left(k_{T 13}^{T C}-k_{T 23}^{T C}\right)^{2}+\left(k_{T 23}^{T C}-1\right)^{2}\left(k_{T 13}^{T C}\right)^{m}+\left(k_{T 23}^{T C}-1\right)^{2}\left(k_{T 23}^{T C}\right)^{m}}}{\left(k_{T 13}^{T C}\right)^{1-\frac{m}{2}}\left(k_{T 23}^{T C}\right)^{\frac{m}{2}}+\left(k_{T 13}^{T C}\right)^{\frac{m}{2}}\left(k_{T 23}^{T C}\right)^{1-\frac{m}{2}}+\left(k_{T 13}^{T C}\right)^{\frac{m}{2}}\left(k_{T 23}^{T C}\right)^{\frac{m}{2}}} \\
& H_{T}^{T E}=\frac{\sqrt{\left(k_{T 13}^{T E}-k_{T 23}^{T E}\right)^{2}+\left(k_{T 23}^{T E}-1\right)^{2}\left(k_{T 13}^{T E}\right)^{m}+\left(k_{T 23}^{T E}-1\right)^{2}\left(k_{T 23}^{T E}\right)^{m}}}{\left(k_{T 13}^{T E}\right)^{1-\frac{m}{2}}\left(k_{T 23}^{T E}\right)^{\frac{m}{2}}+\left(k_{T 13}^{T E}\right)^{\frac{m}{2}}\left(k_{T 23}^{T E}\right)^{1-\frac{m}{2}}+\left(k_{T 13}^{T E}\right)^{\frac{m}{2}}\left(k_{T 23}^{T E}\right)^{\frac{m}{2}}}
\end{aligned}
$$

Where 


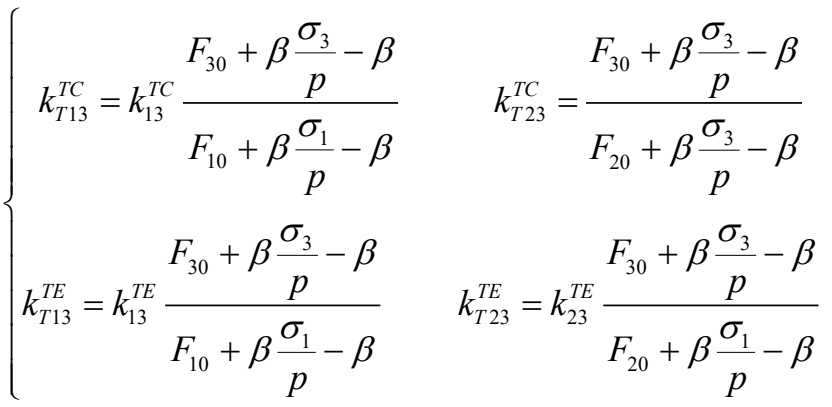

Where $k_{i j}=\sigma_{i} / \sigma_{j}$ is the peak stress ratio, which can be obtained by TC and TE tests. Note that, $k_{23}^{T C}=1$ under TC stress state with $\sigma_{2}=\sigma_{3}$, and $k_{23}^{T E}=k_{13}^{T E}$ under TE stress state with $\sigma_{2}=\sigma_{1}$.

Finally, $m$ can be obtained by combining Eq. (22) and Eq. (23) for $H_{T}^{T C}=H_{T}^{T E}$. Accordingly, the peak contact force ratio $H_{T}$ can be easily calculated from Eq. (21). For example, taking initial fabric $\left.F_{i j}\right|_{0}=1 / 3$, fabric parameter $\beta=0.12$, the friction angle $\varphi_{c r}^{T C}=21.2^{\circ}$ and $\varphi_{c r}^{T E}=20.7^{\circ}$, respectively under TC and TE conditions, the parameter $m$ can be calculated to be equal to 0.420 according to Eq. (22) and Eq. (23) .

\section{PARAMETRIC SENSITIVE ANALYSIS}

The effects of above strength parameters on failure surface of granular material are analyzed in this section. The values of these parameters used in the discussion below are shown in table 1 if not specified.

Table 1: Parameter values for sensitivity analysis

\begin{tabular}{cccccc}
\hline$\beta$ & $\boldsymbol{m}$ & $\boldsymbol{H}_{\boldsymbol{T}}$ & $\left.F_{11}\right|_{0}$ & $\left.F_{22}\right|_{0}$ & $\left.F_{33}\right|_{0}$ \\
\hline 0.1 & 0.5 & 0.2 & $1 / 3$ & $1 / 3$ & $1 / 3$ \\
\hline
\end{tabular}

\subsection{FABRIC PARAMETER $\beta$}

The effect of $\beta$ on the variation of failure envelope in is plotted in Fig.1. As shown in Fig.1, the area of failure envelope increases with the increase of $\beta$, which indicates that the granular medium will possess greater strength as $\beta$ increases because of entailing stronger fabric anisotropy.

Fig. 2 shows the changes of internal friction angle with b-value for different $\beta$ in area I of $\pi$ plane. One can see from Fig. 2 that the internal friction angle first increases and then decreases with the increase of $b$-value, and the ratio of it between $\mathrm{b}=0$ (triaxial compression) and $\mathrm{b}=1$ (triaxial extension) gradually increases. Besides, the internal friction angle increases obviously for a fixed $\mathrm{b}$-value as $\beta$ increases, and the peak point of the curve simultaneously moves left.

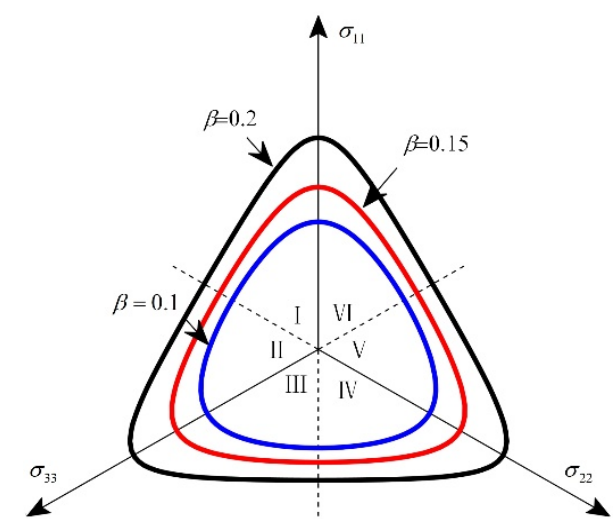

Fig. 1: Failure envelopes with different $\beta$ in $\pi$ plane

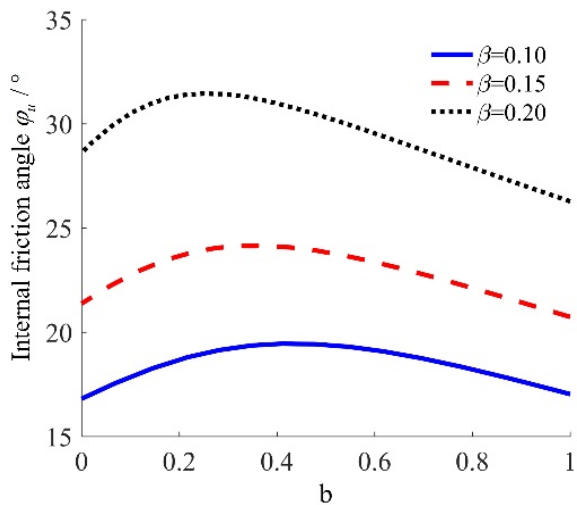

Fig.2: Changes of internal friction angle with $b$ values for different $\beta$ 


\subsection{STRENGTH PARAMETER $m$}

The orientation of failure surface is related to the strength parameter $m$. The proposed anisotropic strength criterion reduces to $\mathrm{M}-\mathrm{N}$ criterion when $m=1$ if the initial anisotropy of granular materials and fabric evolution during loading are ignored. Fig. 3 demonstrates the effect of the variation of $m(=0.1,0.5,1.5)$ on failure envelopes in $\pi$ plane, and the changes of internal friction angle with b-value for different $m$ are also plotted in Fig.4.
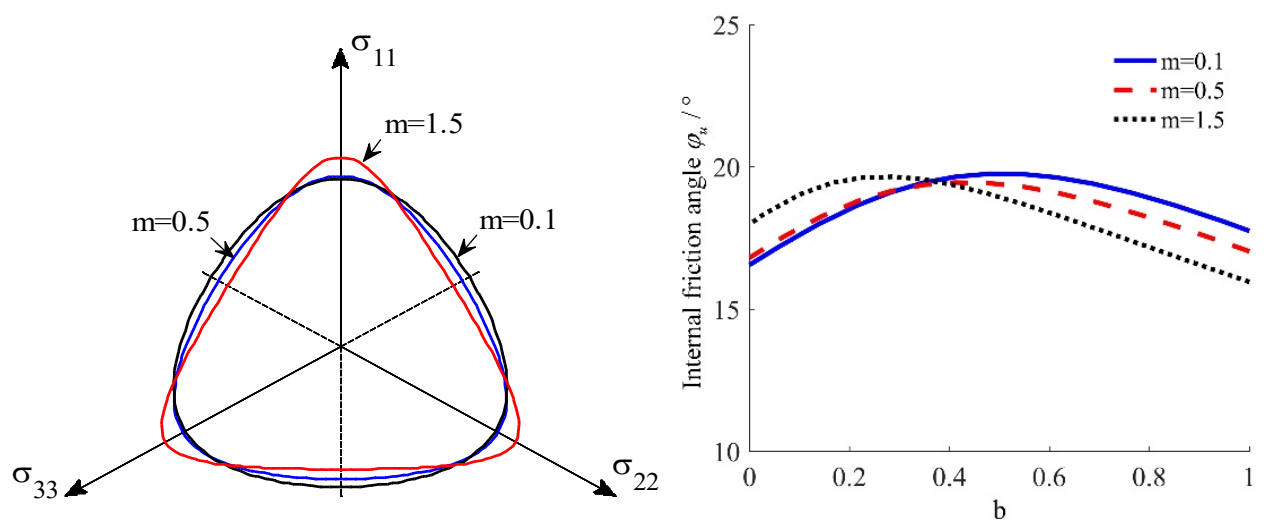

Fig. 3: Failure envelopes with different $m$ in $\pi$ plane

Fig. 4: Changes of internal friction angle with $b$ for different $m$

It can be easily found from Figs. 3 and Fig. 4 that the failure envelope becomes sharper with the increase of $m$, which serves the similar effect as $\beta$. In addition, the ratio of internal friction angles between $b=0$ and $b=1$ also increases with $m$ increasing. However, the internal friction angle gradually increases in triaxial compression condition as $m$ increases, but decreases in triaxial extension condition.

The strength parameter $m$ can be determined by combining the stress ratios of TC to TE conditions as mentioned previously.

\subsection{CONTACT FORCE RATIO $H_{T}$}

Changes of the failure envelope with different $H_{T}(=0.2,0.3,0.4)$ on the premise of ignoring the initial fabric anisotropy are shown in Fig.5. And Fig.6 illustrates the variation of internal friction angle with b-value for different $H_{T}$ accordingly.

As illustrated in Fig.5 and Fig.6, $H_{T}$ plays a similar role as $\beta$. In other word, the greater $H_{T}$, the greater strength, implying that the material can bear a greater tangential contact force under the same normal contact force.
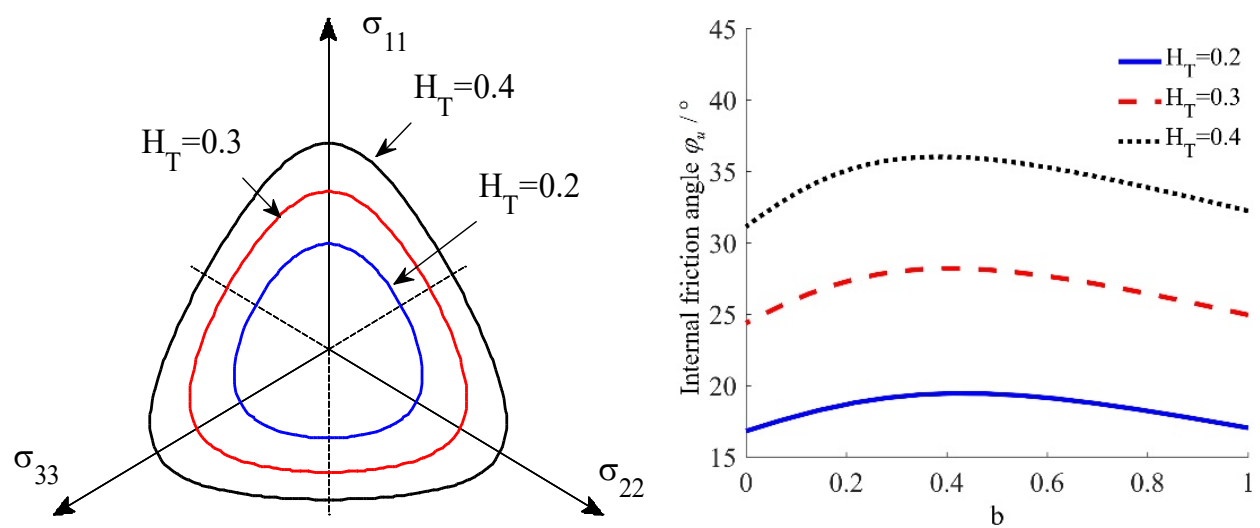

Fig. 5: Failure envelopes with different $H_{T}$ in $\pi$ plane

Fig. 6: Change of internal friction angle with $b$ for different $H_{T}$

\subsection{INITIAL FABRIC ANISOTROPY $\left.F_{i j}\right|_{0}$}

The change of failure envelopes in $\pi$ plane with different initial fabric anisotropy are shown in Fig.7. The shapes of failure envelope clearly illustrate the effect of initial fabric anisotropy, i.e. a biased shape with respect to the pricipal stress axis. With the increase of initial fabric in a certain principal direction, specifically, the triaxial compressive strength 
increases correspondingly in this direction for the greater fabric indicates more contacts, and vice versa.Furthermore, it is interesting that Fig.7(a) will become Fig.7(b) and Fig.7(c) by rotating the failure envelopes for 120 degrees clockwise and counter clockwise around the origin, respectively.

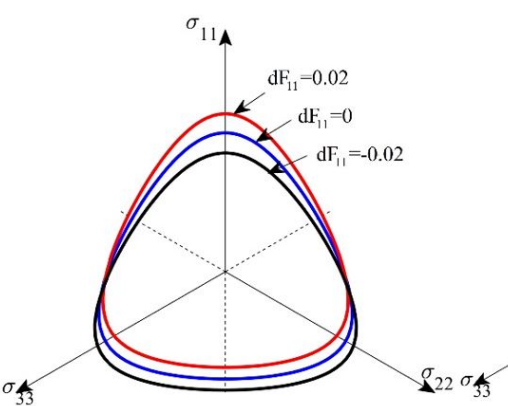

(a)

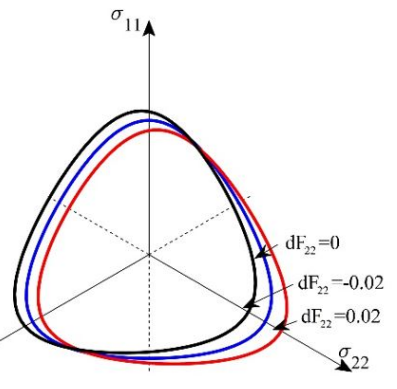

(b)

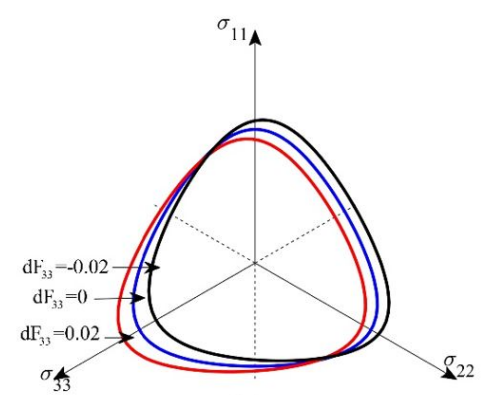

(c)

Fig. 7: Failure envelopes with different initial fabric in $\pi$ plane

Fig.8(a) (c) shows the variation of internal friction angle with b-value for different $F_{11}$ in area I、II and III of $\pi$ plane, respectively. The internal friction angle increases constantly with the increase of $F_{11}$ in area I, but decreases in area III as shown in Fig.8. As for area II, the internal friction angle increases as $F_{11}$ increases when $b=0$, and yet decreases when $b=1$.

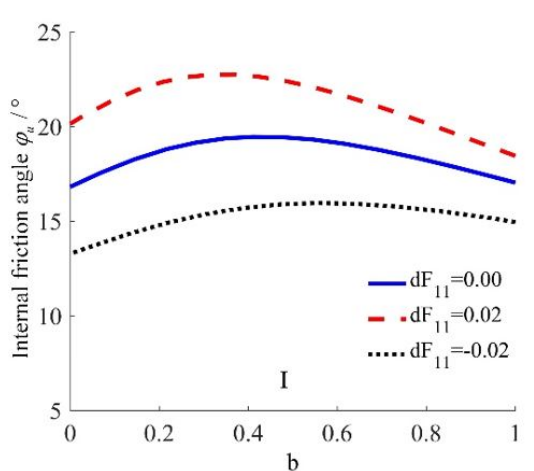

(a)

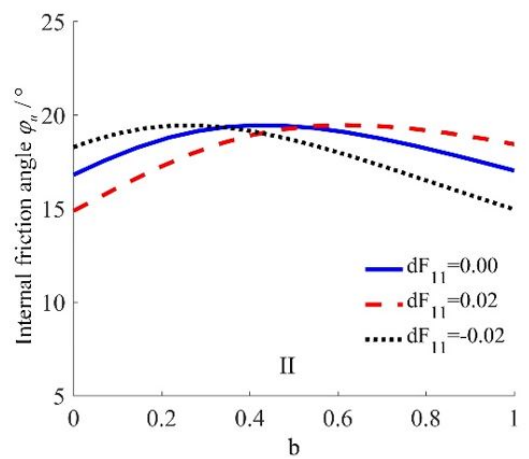

(b)

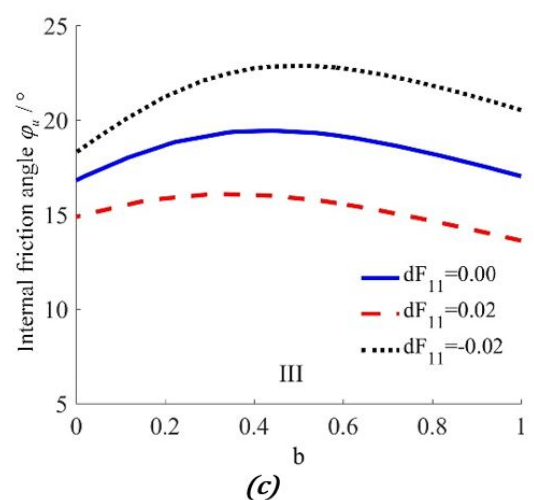

Fig. 8: Change of internal friction angle with $b$ for different initial fabric 


\section{VALIDATION OF THE PROPOSED STRENGTH CRITERION}

\subsection{SANTA MONICA BEACH SAND}

Abelev and Lade (2003) and Abelev et al. (2007) conducted a series of true triaxial drained tests on cubic specimens of Santa Monica Beach sand under confining pressure $\sigma_{3}=50 \mathrm{kPa}$. Due to the lack of specimens' fabric information, the parameter $\beta$ is empirically taken as 0.12 , the initial fabric $\mathrm{F}_{11}=0.3630, \mathrm{~F}_{22}=\mathrm{F}_{33}=0.3185$, and the strength parameter $m=0.033$.

Fig.9 presents the comparison between the experimental results and the predicted results for Santa Monica Beach sand in $\pi$ plane. Meanwhile, the predicted internal friction angles are plotted in Fig.10 together with the test results, where zone I, II and III are different part of $\pi$ plane, as defined in Fig.1.

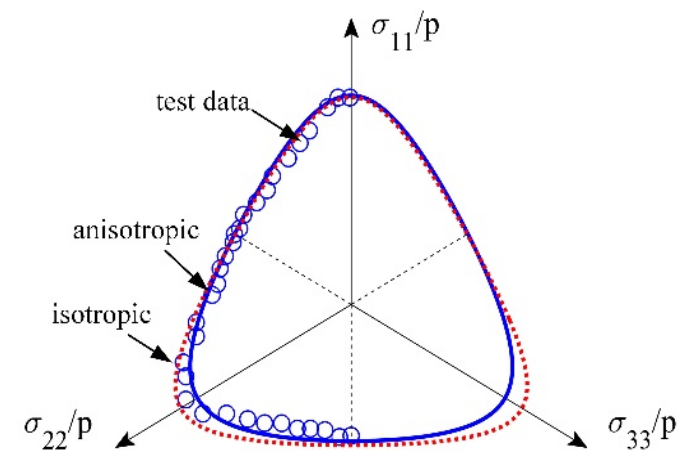

Fig. 9: Predicted results for Santa Monica Beach sand in $\pi$ plane together with the test results

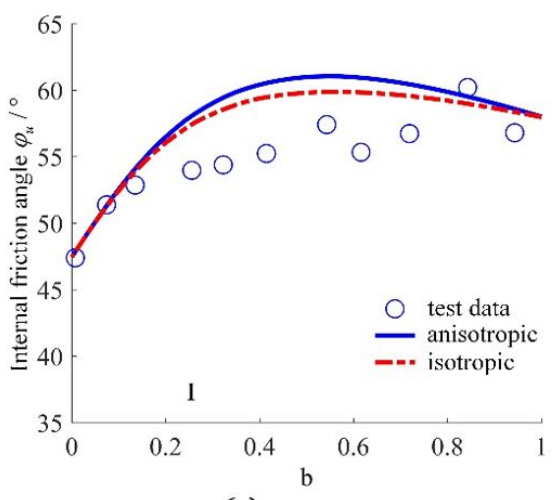

(a)

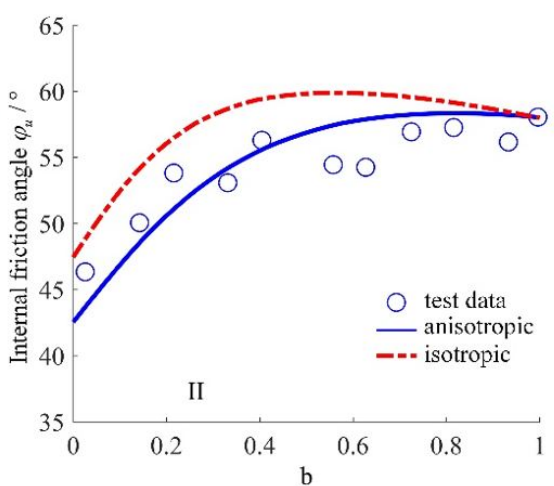

(b)

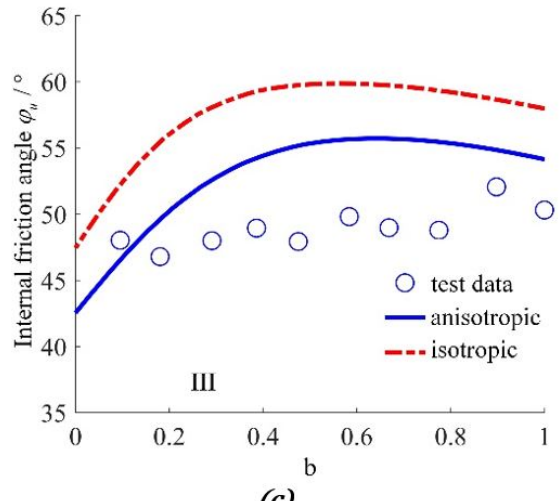

(c)

Fig. 10: Predicted results of internal friction angle with respect to $b$ for Santa Monica Beach sand

One can see from Figs. 9 and Fig.10 that the predicted results from the anisotropic strength criterion give almost the same values as those from the isotropic strength criterion in area I, which is roughly agree with the experimental results. The predicted results from the anisotropic strength criterion are in good agreement with the test data in area II and III. And the isotropic strength criterion makes unsatisfied predictions in area III in that there is strongly initial anisotropy aligning with principal stress axis of the specimens caused by preparation methods. 


\subsection{NEVADA SAND}

Rodriguez and Lade (2013) performed a series of true triaxial tests of cross-anisotropic samples of Nevada sand with different confining pressures. The parameters are chosen as $\beta=0.12, \mathrm{~F}_{11}=0.340, \mathrm{~F}_{22}=\mathrm{F}_{33}=0.330$, and $m=2.27$. Fig.11 shows the predictions and test data of Nevada sand in $\pi$ plane and the comparisons of internal friction angle between measured and predicted results are plotted in Fig.12.

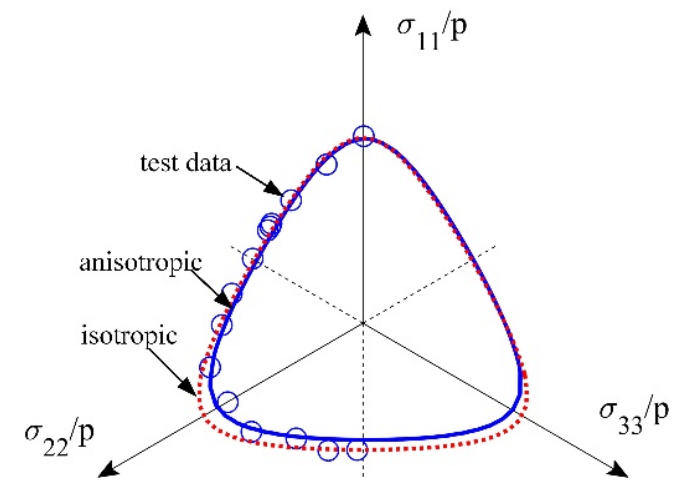

Fig. 11: Predicted results of Nevada sand in $\pi$ plane together with test data

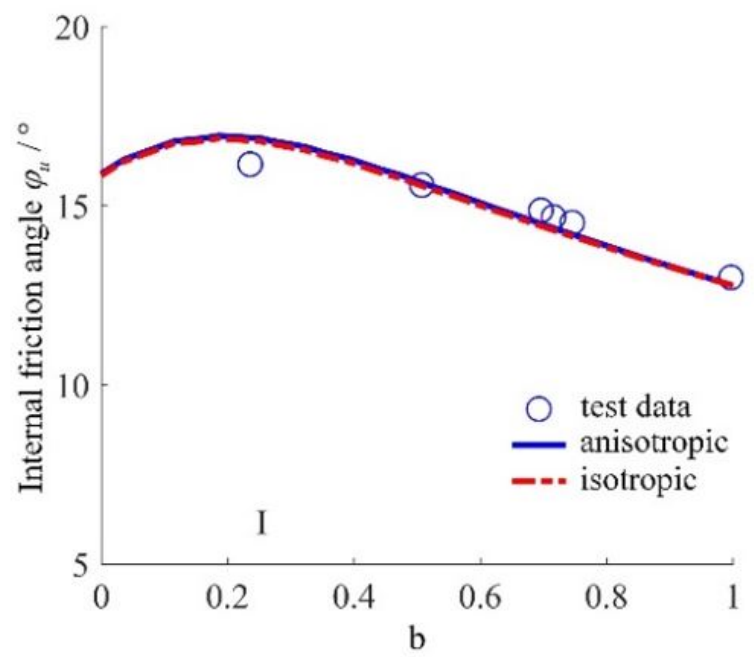

(a)

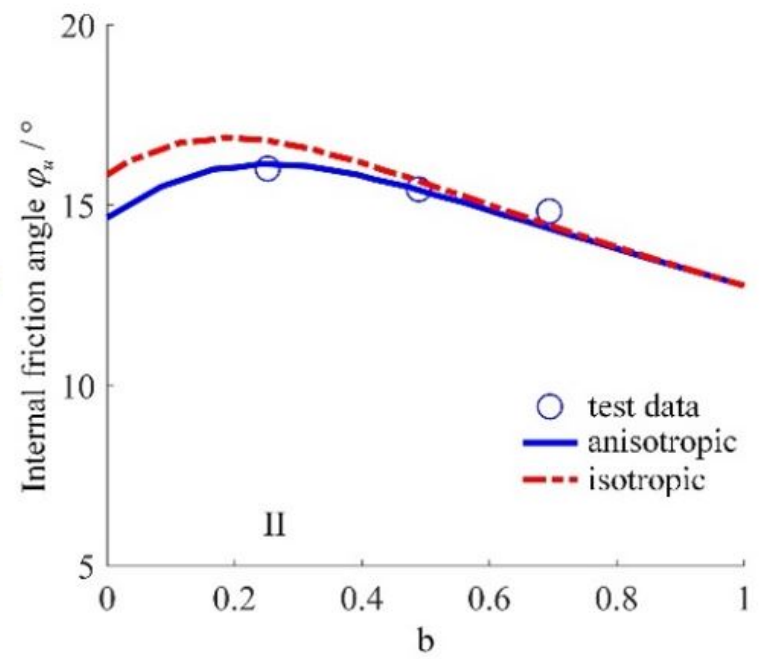

(b)

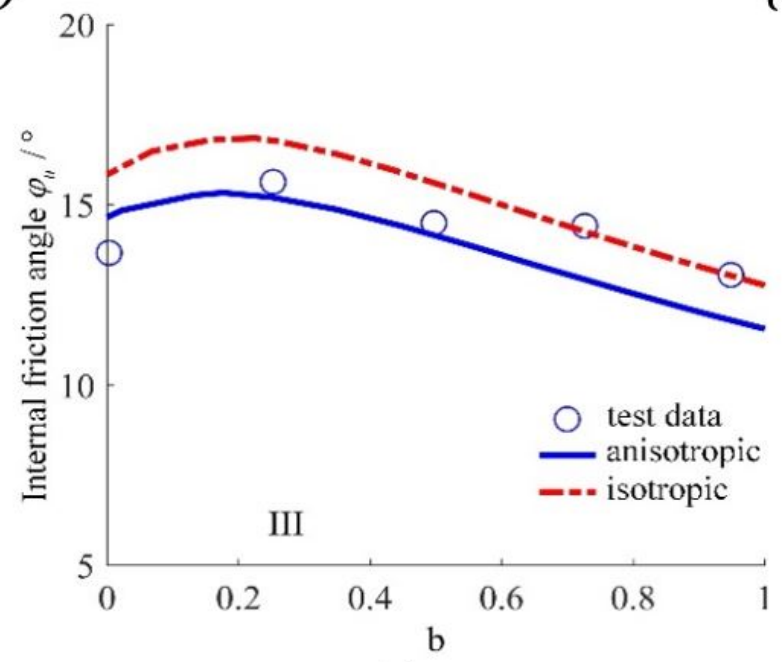

(c)

Fig. 12: Predicted results of internal friction angle for Nevada sand together with test data 
A good consistency can be found in Fig, 11 and Fig. 12 between the measured and predicted results of failure envelope and internal friction angle. The difference between isotropic and anisotropic strength criteria is relatively small for the samples used in tests show a weak fabric anisotropy.

\subsection{CAMBRIA RIVER SAND}

The applicability of the proposed anisotropic strength criterion is also examined by true triaxial tests on Cambria River sand conducted by Ochiai and Lade (1984). The parameters of the samples with weak fabric anisotropy are as follows: i.e. $\beta=0.2, \mathrm{~F}_{11}=0.3430, \mathrm{~F}_{22}=\mathrm{F}_{33}=0.3285$, and $\mathrm{m}=0.142$.

One can see from Fig.13 and Fig.14 that the predicted results capture the experimental trend quite well both in failure envelope and internal friction angle. What is more, the predictions of anisotropic strength criterion show a better agreement with test data than the isotropic one.

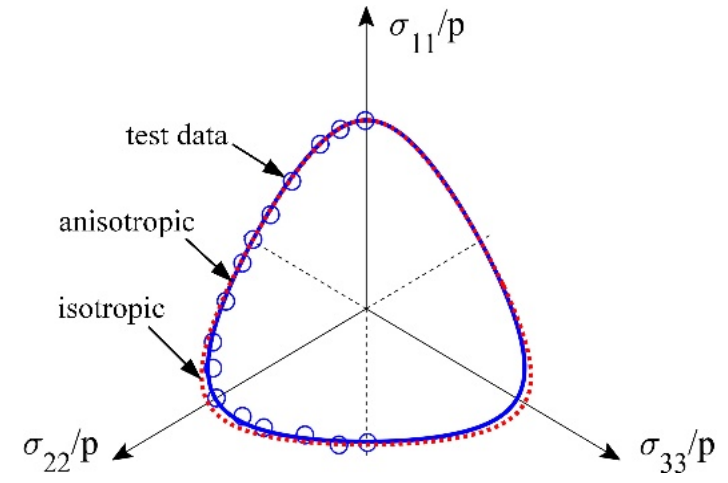

Fig. 13: Predicted results of Cambria River sand in ${ }^{\pi}$ plane together with test data

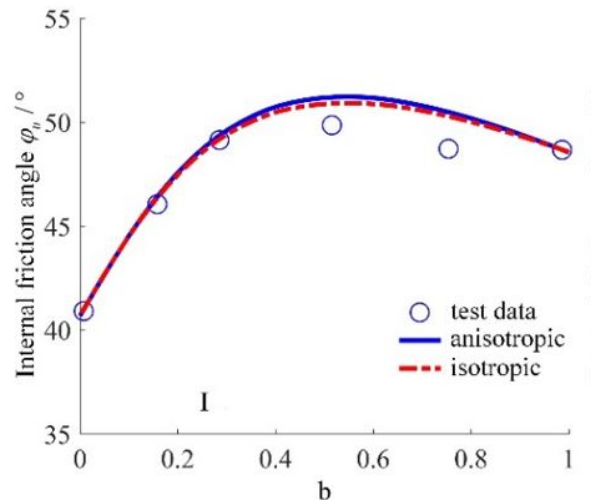

(a)

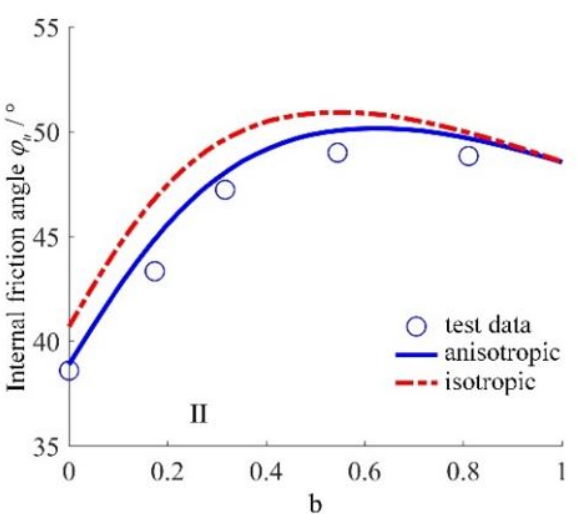

(b)

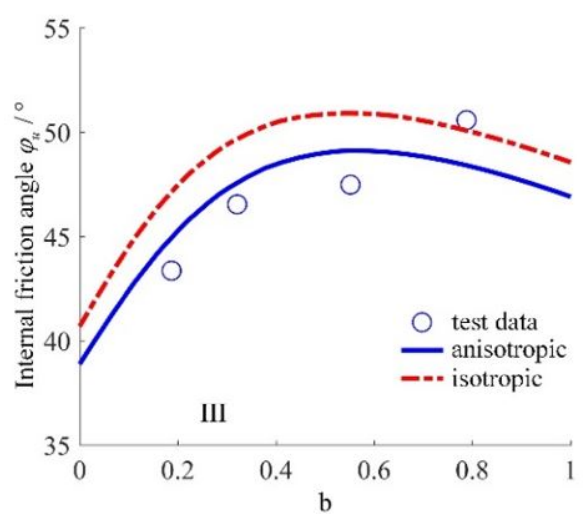

(c)

Fig. 14: Predicted results of internal friction angle with respect to $b$ for Cambria River sand 


\section{CONCLUSIONS}

In this paper, the relationship between macroscopic stress and microscopic contact force of granular material is derived by micromechanical method at first and the new concept of true stress is proposed subsequently. After that, a novel anisotropic strength criterion is proposed for granular medium by introducing the fabric evolution formula into the true stress tensor. The applicability of the proposed anisotropic strength criterion was validated by comparing with experimental results for different granular materials. The preliminary conclusions of the study are as follows:

1. The proposed anisotropic strength criterion incorporates the effect of fabric evolution. Compared with the classical strength criterion, the proposed criterion can embody the effect of not only inherent anisotropy but also the induced anisotropy caused by fabric evolution during loading. It has few parameters with specific physical meaning.

2. The inherent anisotropy of the granular material results in the translation of the failure envelope in $\pi$ plane. Generally speaking, for a certain direction, the stronger the initial fabric, the more the particle contacts, and thus the stronger strength is in this direction.

3. Stress-induced anisotropy has great influence on the strength of granular material. If the other parameters are fixed, the larger the fabric parameter $\beta$, the stronger the stress-induced anisotropy, and the greater the strength ratio of TC to TE condition.

4. The strength ratio of TC to TE condition is determined by the direction of the failure surface of the granular, which reflects the effect of shear modes on material strength. The larger the parameter $\mathrm{m}$, the greater the strength ratio of TC to TE conditions, and the smaller the inclination between the normal of the slip surface and the direction of the major principal stress.

5. The proposed anisotropic strength criterion shows a good agreement with the experimental data of various granular materials, implying it can be utilized to describe the strength feature of anisotropic granular materials.

\section{References}

Abelev A V, Gutta S K, Lade P V, Yamamuro J A. (2007). Modeling cross anisotropy in granular materials. Journal of Engineering Mechanics-ASCE, 133(8):919-932.

Abelev A V, Lade P V. (2003). Effects of cross anisotropy on three-dimensional behavior of Sand. I: Stress-strain behavior and shear banding. Journal of Engineering Mechanics-ASCE, 129(2):160-166.

Cao W, Wang R, Zhang J M (2016) Formulation of anisotropic strength criteria for cohesionless granular materials. Int. J. Geomech., 04016151:1-10.

Chang C S, Gao J. (1996). Kinematic and static hypotheses for constitutive modeling of granulates considering particle rotation. Acta Mech, 115(1/2/3/4):213-229.

Christofferson J, Mehrabadi M M, Nemat-Nassar S. (1981). A micromechanical description on granular material behavior. ASME, Journal of Applied Mechanics, 48:339-344.

Gao Z, Zhao J, Yao Y (2010) A generalized anisotropic failure criterion for geomaterials. International Journal of Solids and Structures, 47(22-23):3166-3185.

Jenkins J T, Strack O D L. (1993). Mean-field inelastic behavior of random arrays of identical spheres. Mechanics of Materials, 16(1-2):25-33.

Kong Y, Zhao J, Yao Y (2013) A failure criterion for cross-anisotropic soils considering microstructure. Acta Geotechnica, 8(6):665-673.

Lade P V (2007) Modeling failure in cross-anisotropic frictional materials. Int. J. Solids Structs 44, No. 16, 5146-5162

Lade P V, Duncan J M. (1975). Elastoplastic stress-strain theory for cohesiveless soil. ASCE, J Geotech Engng Div, 101(10):1037-1053.

Liu Y. (2013). Anisotropic strength criteria of sand: inherent anisotropy. Chinese Journal of Geotechnical Engineering, 35(8):1526-1534.

Liu Y, Chang Ching S., Zhang Duo, et al. (2014). Analytical solution of stress-fabric relationship and failure of granular materials in three dimensions. Chinese Journal of Geotechnical Engineering, 36(3):401-408. 
Lü X L, Huang M S, Andrade, José E (2016) Strength criterion for cross-anisotropic sand under general stress conditions. Acta Geotechnica, 11(6):1339-1350.

Lü X L, Zeng S, Qian J G, Huang M S (2017) DEM analysis of the shear strength of cross-anisotropic sand with non-spherical particles. Geotechnique Letters, 7:1-7.

Matsuoka H, Nakai T. (1974). Stress-deformation and strength characteristics of soil under three different principal stresses. Proc Jpn Soc Civ Engrs, 232:59-70.

Mitchell J K, Soga K. (2005) Fundamentals of soil behavior. Hoboken, NJ: John Wiley \& Sons.

Nemat-Nasser S. (2000) A micromechanically-based constitutive model for frictional deformation of granular materials. Journal of the Mechanics \& Physics of Solids, 48(6):1541-1776.

Ochiai H, Lade P V. (1984). Three-dimensional behavior of sand with anisotropic fabric. Journal of Geotechnical Engineering, 109(10):1313-1328.

Oda M., Koishikawa I, Higuchi T. (1978). Experimental study of anisotropic shear strength of sand by plane strain test. Soils Found, 18(1):25-38.

Pietruszczak S, Mroz Z. (2000). Formulation of anisotropic failure criteria incorporating a microstructure tensor. Computers and Geotechnics, 26(2):105-112.

Rodriguez N M, Lade P V. (2013). True Triaxial Tests on Cross-Anisotropic Deposits of Fine Nevada Sand. International Journal of Geomechanics, 13(6):779-793.

Rothenburg L, Selvadurai A P S. (1981). Micromechanical definitions of the Cauchy stress tensor for particular media. // Selvadurai A P S. (Ed.), Mechanics of Structured Media. Amsterdam, Elsevier, 469-486.

Shao S J, Zhang Y, Chen C L, Shao S. (2015). Strength criterion based on spatially mobilized plane of soils and its comparison with conventional criteria. Chinese Journal of Geotechnical Engineering, 37(4):577-585.

Schweiger H F, Wiltafsky C, Scharinger F, Galavi V. (2009). A multilaminate framework for modelling induced and inherent anisotropy of soils. Geotechnique, 59(2):87-101.

Taylor, D. (1948). Fundamentals of soil mechanics. Chapman and Hall, Limited.; New York.

Tian Y, Yao Y P (2017) A simple method to describe three-dimensional anisotropic failure of soils. Computers \& Geotechnics, 92:210-219.

Tobita Y. (1993). Modified double slip model for anisotropic hardening behaviour of granular materials. Mechanics of Materials, 16(1-2):91-100.

Tobita Y, Yanagisawa E. (1992). Modified stress tensors for anisotropic behavior of granular materials. Soils and Foundations, 32(1):85-99.

Wan R G, Guo J.(2001) Drained cyclic behavior of sand with fabric dependence. Journal of Engineering Mechanics, 127(11):1106-1116.

Xiao Y, Liu H L, Yang G (2012) Formulation of Cross-Anisotropic Failure Criterion for Granular Material. 12(2):182-188.

Yao Y P, Kong Y X. (2012). Study on strength and failure criterion of cross-anisotropic soil. Journal of Hydraulic Engineering, 43(1):43-50.

Yang L T, Li X, Yu H S, Wanatowski D (2015) A laboratory study of anisotropic geomaterials incorporating recent micromechanical understanding. Acta Geotechnica, 11(5):1-19.

Yoshimine M. (2006). Generalized Coulomb's Criterion for 3-dimensional stress conditions. Soils and Foundations, 46(2):259266. 